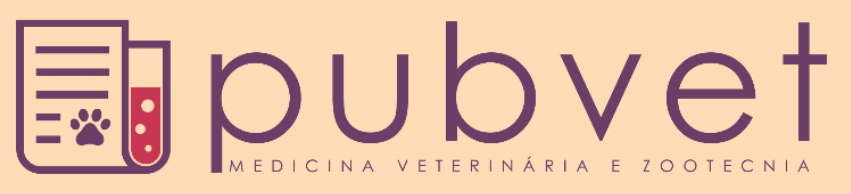

https://doi.org/10.31533/pubvet.v14n11a694.1-8

\title{
Uso da escala de dor da Universidade de Melbourne na avaliação de dois protocolos de analgesia pós-cirúrgica em cadelas submetidas à mastectomia
}

\author{
Thalia Vitoria Mariussi ${ }^{1 *} \bullet$, Maria Cecília de Lima Rorig ${ }^{2} \bullet$, Marcelo Paulino Borges ${ }^{1 \bullet}$, \\ Solimar Dutra da Silveira ${ }^{30}$
}

${ }^{I}$ Discente da Pontifícia Universidade Católica do Paraná, Departamento de Medicina Veterinária. Toledo-PR Brasil.

${ }^{2}$ Médica Veterinária docente da Pontifícia Universidade Católica do Paraná, Departamento de Medicina Veterinária. Toledo -PR Brasil.

${ }^{3}$ Residente em Clínica Cirúrgica de Pequenos Animais da Universidade Federal do Paraná. Palotina - PR Brasil.

*Autor para correspondência: thalia_mariussi@hotmail.com

\begin{abstract}
Resumo. Os tumores mamários em cadelas integram cerca de 52\% das neoplasias em cães. A remoção cirúrgica completa, denominada mastectomia, é considerada o tratamento de eleição. A dor pós-operatória para esse procedimento pode ser classificada de moderada a intensa, exigindo, portanto, um tratamento com fármacos que minimizem a dor. A analgesia preemptiva é um método que consiste na administração de medicamentos antes do estímulo doloroso e dessa forma, tem se destacado entre os protocolos analgésicos. Escalas capazes de avaliar a dor em animais surgiram diante da dificuldade em seu reconhecimento na Medicina Veterinária. A escala de dor da Universidade de Melbourne (UMPS) consiste na avalição dos parâmetros fisiológicos e comportamentais específicos relacionados à dor, aumentando sua sensibilidade e especificidade. Diante disso, o presente estudo teve como objetivo avaliar a eficácia de dois diferentes protocolos de analgesia preemptiva no controle de dor pós-operatória em cadelas submetidas a mastectomia, com a utilização da UMPS. Para isso, a pesquisa contou com 16 pacientes fêmeas que foram aleatoriamente divididas em dois grupos (G1 e G2). O grupo G1 referente ao protocolo de analgesia preemptiva com firocoxibe associado ao adesivo de fentanil trans dérmico, foi composto por 8 fêmeas. Dessa forma, as 8 fêmeas restantes constituíram o grupo G2 recebendo analgesia preemptiva com carprofeno associado ao fentanil trans dérmico. No pós-operatório as pacientes foram avaliadas por meio da aplicação da UMPS em 5 tempos (T1, T2, T3, T12 e T24). O resgate analgésico somente foi efetuado quando a avaliação alcançou pontuação superior a 13. Diante disso, dentre as 16 cadelas que participaram da pesquisa, 11 delas $(68,75 \%)$ demonstraram controle adequado da dor, portanto, não houve a necessidade de resgate, 4 pacientes $(25 \%)$ necessitaram de resgate analgésico e 1 paciente $(6,25 \%)$ foi desclassificada da pesquisa. Contudo, conclui-se que a Escala da Dor da Universidade de Melbourne é um bom método para avaliação do grau de dor em cães e que adesivo de fentanil trans dérmico (Durogesic ${ }^{\circledR}$ ) demonstrou boa atuação no controle da dor com associação a ambos anti-inflamatórios, desde que o mesmo esteja em total aderência com a superfície da pele.
\end{abstract}

Palavras chave: analgesia, dor, mastectomia, resgate analgésico

\section{Use of the University of Melbourne Pain Scale to evaluate two post- surgical analgesia protocols in female dogs undergoing mastectomy}

Abstract. Breast tumors in dogs comprise about 52\% of neoplasms in dogs. Complete surgical removal, called mastectomy, is considered the treatment of choice. Postoperative pain for this procedure can be classified as moderate to severe, therefore requiring treatment 
with drugs that minimize pain. Preemptive analgesia is a method that consists of administering medications before painful stimulus and, thus, has stood out among analgesic protocols. Scales capable of evaluating pain in animals arose in view of the difficulty in its recognition in Veterinary Medicine. The pain scale of the University of Melbourne (UMPS) consists of the assessment of specific physiological and behavioral parameters related to pain, increasing its sensitivity and specificity. Therefore, the present study aimed to evaluate the effectiveness of two different preemptive analgesia protocols in controlling postoperative pain in dogs undergoing mastectomy, using UMPS. For this, the research included 16 female patients who were randomly divided into two groups (G1 and G2). The G1 group regarding the preemptive analgesia protocol with firocoxib associated with the transdermal fentanyl patch, was composed of 8 females. Thus, the remaining 8 females constituted the G2 group receiving preemptive analgesia with carprofen associated with transdermal fentanyl. In the postoperative period, patients were evaluated by applying the UMPS in 5 stages (T1, T2, T3, T12 and T24). The analgesic rescue was only carried out when the evaluation reached a score higher than 13. Therefore, among the 16 female dogs who participated in the research, 11 of them $(68.75 \%)$ demonstrated adequate pain control, therefore, there was no need for rescue, 4 patients $(25 \%)$ required analgesic rescue and 1 patient $(6.25 \%)$ was disqualified from the research. However, it is concluded that the Pain Scale at the University of Melbourne is a good method for assessing the degree of pain in dogs and that transdermal fentanyl patch (Durogesic ${ }^{\circledR}$ ) demonstrated good performance in pain control in association with both anti-inflammatory drugs, as long as it is in total adherence to the skin surface.

Keywords: analgesia, pain, mastectomy, analgesic rescue

\section{Uso de la Escala de dolor de la Universidad de Melbourne para evaluar dos protocolos de analgesia posquirúrgica en perras sometidas a mastectomía}

Resumen. Los tumores de mama en perros comprenden aproximadamente el $52 \%$ de las neoplasias en perros. La extirpación quirúrgica completa, llamada mastectomía, se considera el tratamiento de elección. El dolor postoperatorio para este procedimiento puede clasificarse como moderado a severo, por lo que requiere tratamiento con medicamentos que minimicen el dolor. La analgesia preventiva es un método que consiste en administrar medicamentos antes del estímulo doloroso y, por lo tanto, se ha destacado entre los protocolos analgésicos. Las escalas capaces de evaluar el dolor en animales surgieron en vista de la dificultad en su reconocimiento en Medicina Veterinaria. La escala de dolor de la Universidad de Melbourne (UMPS) consiste en la evaluación de parámetros fisiológicos y de comportamiento específicos relacionados con el dolor, aumentando su sensibilidad y especificidad. Por lo tanto, el presente estudio tuvo como objetivo evaluar la efectividad de dos protocolos de analgesia preventiva diferentes para controlar el dolor postoperatorio en perros sometidos a mastectomía, utilizando UMPS. Para esto, la investigación incluyó a 16 pacientes hembras que fueron divididas aleatoriamente en dos grupos (G1 y G2). El grupo G1 con respecto al protocolo de analgesia preventiva con firocoxib asociado con el parche transdérmico de fentanilo, estaba compuesto por 8 hembras. Por lo tanto, las 8 hembras restantes constituyeron el grupo G2 que recibió analgesia preventiva con carprofeno asociado con fentanilo transdérmico. En el postoperatorio, los pacientes fueron evaluados aplicando el UMPS en 5 tiempos (T1, T2, T3, T12 y T24). El rescate analgésico solo se realizó cuando la evaluación alcanzó una puntuación superior a 13. Por lo tanto, entre las 16 perras que participaron en la investigación, 11 de ellas $(68,75 \%)$ demostraron un control adecuado del dolor, por lo tanto, no hubo necesidad de rescate, 4 pacientes (25\%) requirieron rescate analgésico y 1 paciente $(6,25 \%)$ fue descalificado de la investigación. Sin embargo, se concluye que la Escala de dolor en la Universidad de Melbourne es un buen método para evaluar el grado de dolor en perros y que el parche transdérmico de 
fentanilo (Durogesic $®$ ) demostró un buen desempeño en el control del dolor en asociación con ambos fármacos antiinflamatorios, siempre y cuando esté en total adherencia a la superficie de la piel.

Palabras clave: analgesia, dolor, mastectomía, rescate analgésico

\section{Introdução}

Os tumores mamários em cadelas constituem cerca de 52\% de todas as neoplasias em cães, e aproximadamente 50\% desses são de característica maligna. A remoção cirúrgica completa, denominada mastectomia, com amplas margens de segurança é o tratamento de escolha, exceto para animais com diagnóstico de carcinoma inflamatório ou com a presença de metástases à distância. A dor decorrente desse procedimento já foi demonstrada em diversos estudos e pode ser classificada de moderada a intensa (Fossum, 2014).

Atualmente, a preocupação sobre a eficácia dos analgésicos, seus efeitos adversos sistêmicos e ainda o risco do seu uso abusivo, culminaram com a necessidade de procurar alternativas mais eficazes no controle da dor na medicina veterinária (Gaynor \& Muir, 2009). A analgesia preemptiva é um dos métodos que tem se destacado entre os protocolos analgésicos direcionados ao controle da dor, essa consiste na administração de fármacos previamente ao estímulo doloroso, com o intuito de reduzir as doses das drogas necessárias para o alívio da dor, quando comparada ao uso de analgésicos somente após o estímulo doloroso (Wetmore \& Glowski, 2000).

A escala de dor da Universidade de Melbourne (UMPS) consiste na avalição dos parâmetros fisiológicos e comportamentais relacionados à dor, e dessa forma aumenta a sua sensibilidade e especificidade. Inclui descrições múltiplas em seis categorias, sendo o animal avaliado em cada parâmetro com atribuição de uma pontuação de zero (sem dor) a três (dor máxima), pontuando no máximo 27 pontos, o que indica a maior experiência dolorosa possível (Gaynor \& Muir, 2009).

Assim, o presente estudo teve como propósito avaliar a eficácia de dois diferentes protocolos de analgesia preemptiva no controle de dor pós-operatória em cadelas submetidas a mastectomia por meio da Escala de dor da Universidade de Melbourne.

\section{Material e métodos}

O projeto foi aprovado pelo Comitê de ética no Uso de Animais - CEUA da PUCPR e obteve protocolo 01215. Além disso, teve como local de desenvolvimento a Clínica Veterinária Universitária - CLIVET da Pontifícia Universidade Católica do Paraná, campus Toledo e a Clínica Veterinária Aukmia, localizada na Rua Almirante Barroso, 2546, Centro de Toledo, Paraná.

Durante o estudo foram avaliadas 16 cadelas aleatoriamente divididas em dois grupos (G1 e G2), cuja abordagem terapêutica foi a cirurgia de mastectomia. Diante disso, oito fêmeas participaram do grupo G1 $(\mathrm{n}=8)$ e receberam analgesia preemptiva com firocoxibe na dose de $5 \mathrm{mg} / \mathrm{kg}$ (Viana, 2014), associado ao adesivo de fentanil trans dérmico (concentração de 25, 50, 75 ou $100 \mu \mathrm{g} /$ hora) de acordo com o peso de cada paciente ( $25 \mu \mathrm{g} / \mathrm{h}$ para cães de 5 a $10 \mathrm{~kg}$ e para cães entre 10 e $20 \mathrm{~kg}, 50 \mu \mathrm{g} / \mathrm{h}$ ) (Pascoe, 2000). O segundo grupo G2 $(\mathrm{n}=8)$ foi constituído por outras 8 fêmeas que receberam analgesia preemptiva com carprofeno na dose de $4,4 \mathrm{mg} / \mathrm{kg}$ (Viana, 2014) associado ao fentanil trans dérmico nas mesmas recomendações supracitadas.

As pacientes foram internadas 24 horas prévias ao procedimento cirúrgico, iniciando a preparação por meio de tricotomia em região cervico-torácica ou torácica (Figura 1) e fixação do adesivo de fentanil trans dérmico (Durogesic ${ }^{\circledR}$ ) de acordo com o peso corporal de cada paciente, como indicado por Fantoni (2012) (Figura 2). Para a garantia da completa fixação do adesivo e a fim de evitar sua remoção ou qualquer incidente as pacientes foram devidamente enfaixadas com atadura e esparadrapo (Figura 3). Também foi administrado neste mesmo momento o antinflamatório não esteroidal, por via oral, e realizado exame físico para a avaliação dos parâmetros vitais de cada paciente.

O protocolo anestésico foi composto por medicação pré-anestésica com acepromazina na dose de $0,05 \mathrm{mg} / \mathrm{kg}$ por via intramuscular, após 20 minutos, indução com propofol na dose de $4 \mathrm{mg} / \mathrm{kg}$ e manutenção com sevoflurano (conectado a uma fonte de oxigênio a 100\% (Figura 4). Quando 
necessário, durante o transoperatório, foi realizado resgate analgésico pela aplicação de fentanil na dose de $0,02 \mathrm{mg} / \mathrm{kg}$ pela via endovenosa (Massone, 1999).

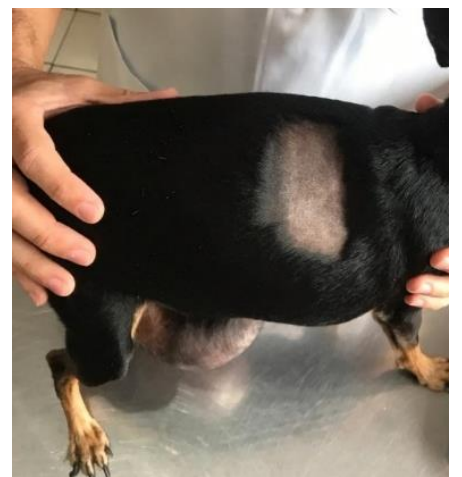

Figura 1. Tricotomia em região torácica

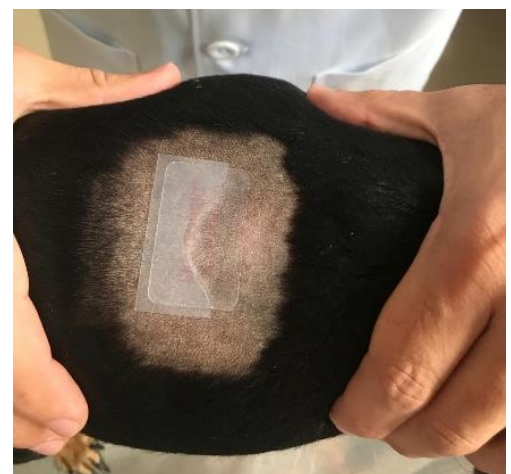

Figura 2. Adesivo de fentanil transdérmico fixado (Durogesic $®)$.

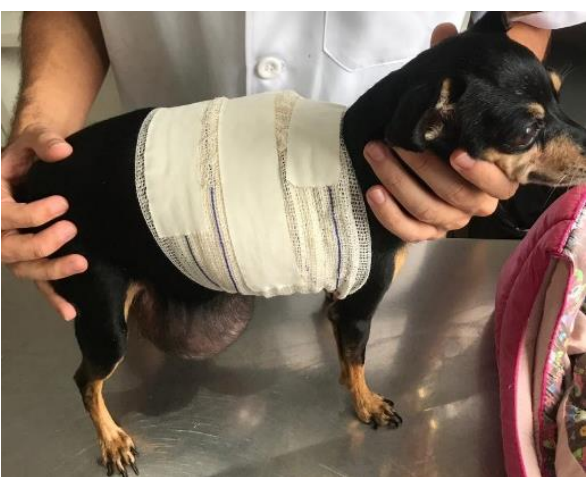

Figura 3. Fixação com atadura e esparadrapo.

Após o término da intervenção cirúrgica, as fêmeas foram mantidas em ambiente tranquilo para a avaliação da dor, por meio da aplicação da Escala de dor da Universidade de Melbourne (Figura 5). Os animais foram avaliados quanto a alterações comportamentais como postura, resposta a palpação, vocalização, estado mental e grau de atividade, não descartando parâmetros fisiológicos como frequência cardíaca, frequência respiratória, temperatura retal dilatação pupilar e salivação. A partir disso foram atribuídas pontuações em cada categoria gerando um escore final por meio da soma de todas categorias, a qual pode variar de 0 a 27 (Firth \& Haldane, 1999). De acordo com a pontuação obtida na escala de dor, o resgate analgésico somente foi realizado quando se obteve graduação superior a 13 pontos, utilizando-se metadona na dose de $0,2 \mathrm{mg} / \mathrm{kg}$ por via intravenosa lenta como forma de recuperação analgésica (Viana, 2014).

As fêmeas foram avaliadas a partir do término da cirurgia e recuperação anestésica, sendo a escala aplicada em cinco tempos (T1, T2, T3, T12 e T24) que correspondem respectivamente 1 hora, 2 horas, 3 horas, 15 horas e 24 horas após o término da intervenção cirúrgica.

Após a última avaliação pela Escala de Melbourne, o adesivo de fentanil trans dérmico foi removido. As pacientes receberam alta com prescrição de tramadol na dose de $4 \mathrm{mg} / \mathrm{kg}$ a cada 12 horas e manutenção do antinflamatório utilizado no respectivo grupo durante 5 dias, além de amoxicilina + clavulanato de potássio, dipirona sódica, associado a cuidados com a ferida cirúrgica.

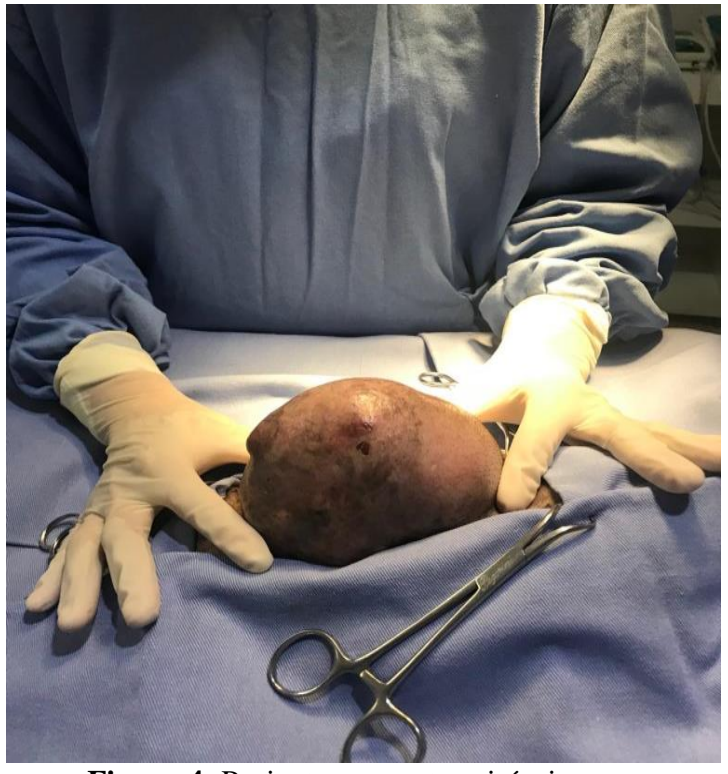

Figura 4. Paciente em centro cirúrgico para realização da mastectomia

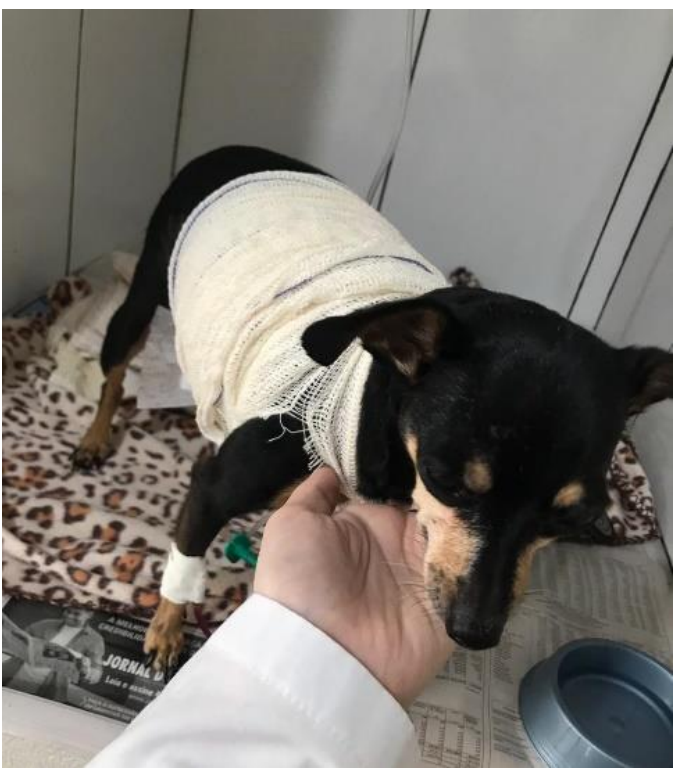

Figura 1. Paciente após o término do procedimento cirúrgico (T1). 


\section{Resultados}

Entre as 16 cadelas que participaram da pesquisa, 11 delas (68,7\%) demonstraram controle adequado da dor, visto que não atingiram o escore compatível com a dor pela UMPS. Além disso, as pacientes já conseguiam caminhar e se alimentar horas após o término do procedimento e mostravam-se indiferentes à palpação da ferida cirúrgica, sendo assim, nesses casos não houve necessidade de proceder o resgate analgésico.

Em ambos os grupos (G1 e G2) houve a necessidade de resgate analgésico em duas pacientes (G1 n1; G1 n5 \& G2 n2; G2 n3) conforme apresentado na (Tabela 1). Dessa forma, o resgate em (G1 n1 \& G2 n2) foi realizado com aplicação de fentanil $(0,02 \mathrm{mg} / \mathrm{kg}$, IV) no transoperatório devido a observação do aumento da frequência cardíaca, frequência respiratória e dilatação pupilar indicando que as pacientes poderiam estar sentindo dor. Entretanto, após a aplicação ambas tiveram uma melhora progressiva e mantiveram-se estáveis durante as cinco avaliações pós-operatórias pela UMPS sem pontuação compatível com a dor. O resgate em (G1 n5 \& e G2 n3) foi realizado com metadona $(0,2 \mathrm{mg} / \mathrm{Kg}$, IV), visto que a avaliação das pacientes no pós-operatório atingiu escore compatível com a dor através da UMPS. Todavia após o resgate analgésico foi possível perceber melhora gradativa em ambas, até a voltarem ao seu estado normal.

Além disso, uma paciente do grupo G2 (G2 n8) foi desclassificada do protocolo proposto na presente pesquisa, pois quatro horas após a aplicação do adesivo de fentanil e administração do anti-inflamatório firocoxibe o animal apresentou-se agitado e desconfortável, então optou-se pela remoção do adesivo e a paciente prosseguiu o tratamento com outro protocolo estabelecido pelo Médico Veterinário responsável.

Diante do exposto, a presente pesquisa atingiu um porcentual de 68,75\% (Figura 6) pacientes que obtiveram boa atuação no controle da dor, não necessitando de resgate analgésico. Ainda, em $25 \%$ dos animais houve a necessidade de resgate analgésico, sendo que $12,5 \%$ desses, foi realizado no transoperatório com fentanil e os outros $12,5 \%$ no pós-operatório com administração da metadona. A pesquisa obteve uma paciente desclassificada $(6,25 \%)$. Na correlação entre os grupos (G1 e G2) não houve diferença significativa, visto que em ambos os grupos $25 \%$ das pacientes precisaram de resgate analgésico, demonstrando exceto nos casos onde houve desprendimento do adesivo de fentanil, os dois protocolos obtiveram boa atuação no controle da dor pós-operatória (Tabela 1).

Tabela 1. Relação de pacientes de cada grupo (G1 ou G2) com seus respectivos protocolos (Carprofeno ou Firocoxibe) avaliadas na pesquisa, incluindo a necessidade ou não, de resgate analgésico (metadona ou fentanil), e a relação com a aderência do adesivo de Fentanil.

\begin{tabular}{|c|c|c|c|c|c|}
\hline Paciente & AINE & Resgate Metadona & Resgate Fentanil & Excluído do protocolo & Desprendimento do adesivo \\
\hline (G1 n1) & Carprofeno & $\mathrm{N}$ & $\mathrm{S}$ & $\mathrm{N}$ & $\mathrm{N}$ \\
\hline (G1 n2) & Carprofeno & $\mathrm{N}$ & $\mathrm{N}$ & $\mathrm{N}$ & $\mathrm{N}$ \\
\hline (G1 n3) & Carprofeno & $\mathrm{N}$ & $\mathrm{N}$ & $\mathrm{N}$ & $\mathrm{N}$ \\
\hline (G1 n4) & Carprofeno & $\mathrm{N}$ & $\mathrm{N}$ & $\mathrm{N}$ & $\mathrm{N}$ \\
\hline (G1 n5) & Carprofeno & $S$ & $\mathrm{~N}$ & $\mathrm{~N}$ & S \\
\hline (G1 n6) & Carprofeno & $\mathrm{N}$ & $\mathrm{N}$ & $\mathrm{N}$ & $\mathrm{N}$ \\
\hline (G1 n7) & Carprofeno & $\mathrm{N}$ & $\mathrm{N}$ & $\mathrm{N}$ & $\mathrm{N}$ \\
\hline (G1 n8) & Carprofeno & $\mathrm{N}$ & $\mathrm{N}$ & $\mathrm{N}$ & $\mathrm{N}$ \\
\hline (G2 n1) & Firocoxibe & $\mathrm{N}$ & $\mathrm{N}$ & $\mathrm{N}$ & $\mathrm{N}$ \\
\hline (G2 n2) & Firocoxibe & $\mathrm{N}$ & $S$ & $\mathrm{~N}$ & $\mathrm{~N}$ \\
\hline (G2 n3) & Firocoxibe & $S$ & $\mathrm{~N}$ & $\mathrm{~N}$ & $S$ \\
\hline (G2 n4) & Firocoxibe & $\mathrm{N}$ & $\mathrm{N}$ & $\mathrm{N}$ & $\mathrm{N}$ \\
\hline (G2 n5) & Firocoxibe & $\mathrm{N}$ & $\mathrm{N}$ & $\mathrm{N}$ & $\mathrm{N}$ \\
\hline (G2 n6) & Firocoxibe & $\mathrm{N}$ & $\mathrm{N}$ & $\mathrm{N}$ & $\mathrm{N}$ \\
\hline (G2 n7) & Firocoxibe & $\mathrm{N}$ & $\mathrm{N}$ & $\mathrm{N}$ & $\mathrm{N}$ \\
\hline (G2 n8) & Firocoxibe & - & - & S & - \\
\hline
\end{tabular}

Nota: (G) indica o grupo pertencente e (n) o número da amostra de cada grupo, as letras $\mathrm{N} \mathrm{e} \mathrm{S}$ correspondem respectivamente a não e sim. 


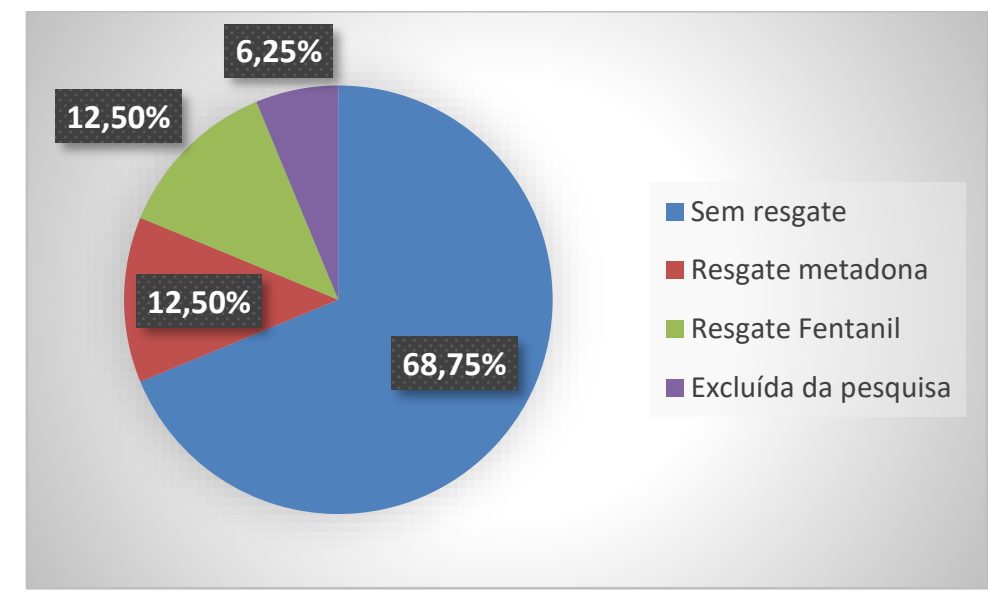

Figura 6. Relação em porcentagem da necessidade de resgate analgésico (metadona ou fentanil) bem como da desclassificação de pacientes.

\section{Discussão}

Em conformidade com estudos de Aleixo et al. (2004) e Gaynor \& Muir (2009) o adesivo de fentanil trans dérmico (Durogesic ${ }_{\circledast}$ ) demonstrou boa atuação no controle da dor juntamente com o antiinflamatório carprofeno ou firocoxibe, uma vez que, dos 15 animais avaliados, 11 deles não ultrapassaram a pontuação compatível com a dor para a Escala de Dor da Universidade de Melbourne, não necessitando, dessa forma, de resgate analgésico.

Aleixo et al. (2004) ressaltam em sua pesquisa a indispensabilidade de uma boa higienização da área de fixação do adesivo, incluindo nisso uma tricotomia adequada. Dessa forma, considera-se que a necessidade do resgate analgésico em uma paciente do grupo G1 (G1 n5) seja consequência de uma falha na tricotomia, visto que um defeito na lâmina da máquina fez com que ainda restassem pelos na região da fixação do adesivo. Devido essa adversidade, considera-se que o adesivo não tenha entrado em total contato com a pele facilitando seu desprendimento, e dessa forma, comprometendo a absorção do princípio ativo, consequentemente o controle da dor. Entretanto, levando em consideração que as concentrações séricas após a retirada do adesivo diminuem de forma gradativa (Durogesic ${ }^{\circledR}$ D-Trans) e tendo em vista que durante as primeiras avaliações a paciente apresentava-se estável e sem sinais de dor, conclui-se que o tempo que o adesivo permaneceu fixado foi suficiente para uma liberação considerável de medicamento, de tal maneira que houve o controle da dor até o momento da terceira avaliação (T3).

Conforme as orientações da bula de Durogesic ${ }^{\circledR}$ D-Trans é importante a frequente monitoração da adesão do medicamento a pele, destacando ainda, que há a possibilidade de o adesivo não aderir satisfatoriamente a todos os pacientes. Portanto, correlaciona-se isso à necessidade do resgate em uma paciente do grupo G2 (G2 n3), a qual demonstrou alteração nos parâmetros vitais com aumento da pontuação da escala, diante disso, ao verificar o adesivo, constatou-se que o mesmo não estava totalmente aderido à superfície da pele devido ás dobras cutâneas provenientes da paciente, que apresentava-se acima do peso. Ainda de acordo com a bula do fentanil transdérmico, esse medicamento pode ser passível de efeitos adversos como depressão, aumento de frequência cardíaca, espasmos musculares, alucinação e euforia (Durogesic ${ }^{\circledR} \mathrm{D}$-Trans). Dessa forma acredita-se que a manifestação de desconforto e agitação do animal desclassificado (G2 n8) tenha sido proveniente das reações adversas do fentanil trans dérmico.

Na fisiopatologia da dor há a ativação do sistema nervoso autônomo simpático a partir da liberação das catecolaminas, resultando em estresse, aumento da frequência cardíaca, frequência respiratória, pressão arterial, arritmias, salivação, midríase e sudorese (Aleixo et al., 2017). Quando prolongada, essa ativação pode gerar efeitos prejudiciais para o organismo como a má perfusão regional e comprometimento do funcionamento dos órgãos vitais (Faleiros et al., 1997). Portanto, quando foi observado o aumento de frequência cardíaca, frequência respiratória e midríase durante o transoperatório, optou-se pela aplicação do fentanil, pressupondo que as pacientes (G1n1 e G2n2) poderiam estar sentindo dor. 
Aleixo et al. (2017) esclarece a importância do cuidado especial em pacientes com dor, já que essa está relacionada também com a ativação do sistema neuroendócrino. Esse sistema quando ativado aumenta os níveis de hormônios catabólicos (catecolaminas, cortisol, glucagon e hormônio do crescimento) e promove a diminuição de mediadores anabólicos (testosterona e insulina) (Faleiros et al., 1997). Diante disso, a elevada concentração de cortisol, promove um aumento do catabolismo de proteínas, da concentração de glicose sanguínea e imunossupressão, resultando no desequilíbrio de fluidos (Basso et al., 2008).

Dentre as alterações provocadas pelo processo da dor é necessário mencionar ainda, o aumento dos riscos a infecções, o retardo na cicatrização e a diminuição da ingestão hídrica e de alimentos. Também, modificações nos sistemas cardiorrespiratórios como arritmias ventriculares e digestório como atonia, hipotonia e tendência a ulcerações, aumentando ainda mais o tempo de recuperação desses animais (Barratt, 1997; Tranquilli et al., 2013). Portanto, se não houver a correta identificação e controle da dor, a homeostase é superada por respostas endócrinas e pelo estresse, podendo provocar automutilação, imunossupressão e até mesmo óbito do animal (Gaynor \& Muir, 2009).

\section{Conclusão}

Diante dos fundamentos expostos, é possível concluir que a Escala da Dor da Universidade de Melbourne é um bom método para avaliação do grau da dor em cães. Além disso, o adesivo de fentanil trans dérmico (Durogesic ${ }^{\circledR}$ ) demonstrou boa atuação no controle da dor juntamente com a associação com o anti-inflamatório Carprofeno e com o Firocoxibe, ou seja, os dois protocolos são eficientes. Entretanto, é de extrema importância a garantia de que o adesivo de fentanil esteja em total aderência com a superfície da pele.

\section{Referências bibliográficas}

Aleixo, G A S, Ribeiro, V. M., Maciel Junior, B. S., Sousa, A. A., \& Oliveira, C. R. (2004). Uso do analgésico fentanil transdérmico em um cão. Ciência Veterinária Tropical, 7(2/3), 140-144.

Aleixo, Grazielle Anahy Sousa, Tudury, E. A., Coelho, M. C. O. C., Andrade, L. S. S., \& Bessa, A. L. N. G. (2017). Tratamento da dor em pequenos animais: classificação, indicações e vias de administração dos analgésicos (revisão de literatura: parte II). Medicina Veterinária, 11(1), 29-40. DOI: https://doi.org/10.26605/medvet-n1-1596

Barratt, S. M. (1997). Advances in acute pain management. International Anesthesiology Clinics, 35(2), 27-48. DOI: https://doi.org/10.1097/00004311-199703520-00004

Basso, P. C., Raiser, A. G., Carregaro, A. B., \& Muller, D. C. M. (2008). Analgesia transoperatória em cães e gatos. Clínica Veterinária, 77, 62-68.

Faleiros, R. R., Alves, G. E. S., \& Marques Júnior, A. P. (1997). Dor aguda: vias anatômicas, bioquímicas e fisiopatologia. Caderno Técnico Da Escola Veterinária Da UFMG, 21, 5-14.

Fantoni, D. (2012). Tratamento da dor na clínica de pequenos animais. Elsevier Brasil.

Firth, A. M., \& Haldane, S. L. (1999). Development of a scale to evaluate postoperative pain in dogs. Journal of the American Veterinary Medical Association, 214(5), 651-659.

Fossum, T. W. (2014). Cirurgia de pequenos animais (4th ed., Vol. 1). Elsevier Brasil.

Gaynor, J. S., \& Muir, W. W. (2009). Manual de controle da dor em medicina veterinária (Vol. 1). MedVet. Massone, F. (1999). Anestesiologia veterinária. In Farmacologia e técnicas. Guanabara Koogan.

Pascoe, P. J. (2000). Opioid analgesics. Veterinary Clinics: Small Animal Practice, 30(4), 757-772. DOI: https://doi.org/10.1016/s0195-5616(08)70005-6

Tranquilli, W. J., Thurmon, J. C., \& Grimm, K. A. (2013). Lumb and Jones' veterinary anesthesia and analgesia. John Wiley \& Sons.

Viana, F. A. B. (2014). Guia terapêutico veterinário. In Lagoa Santa.

Wetmore, L. A., \& Glowski, M. M. (2000). Epidural analgesia in veterinary critical care. Clinical Techniques in Small Animal Practice, 15(3), 177-188. DOI: https://doi.org/10.1053/svms.2000.16545

Recebido: 17 de junho, 2020

Aprovado: 5 de agosto, 2020.

Disponível online: 23 de outubro, 2020
Licenciamento: Este artigo é publicado na modalidade Acesso Aberto sob a licença Creative Commons Atribuição 4.0 (CC-BY 4.0), a qual permite uso irrestrito, distribuição, reprodução em qualquer meio, desde que $o$ autor e a fonte sejam devidamente creditados. 
ANEXO 1 - ESCALA DE MELBOURNE

Escala de dor da Universidade de Melbourne

QUADRO 4 - Escala de Dor da Universidade de Melbourne.

\begin{tabular}{|c|c|c|}
\hline Varlível & Critério & Escore \\
\hline \multicolumn{3}{|l|}{ Dados fislologicos } \\
\hline 8. & $\begin{array}{l}\text { Dados fisioidgicos dentro dos valores de } \\
\text { referencia }\end{array}$ & 0 \\
\hline \multirow{4}{*}{$\begin{array}{l}\text { b. Escolha apenas } \\
\text { um: }\end{array}$} & Pupilas dilatadas & 2 \\
\hline & $>20 \%$ & 1 \\
\hline & $>50 \%$ & 2 \\
\hline & $>100 \%$ & 3 \\
\hline \multicolumn{3}{|l|}{$\begin{array}{l}\text { d. Escoina apenas } \\
\text { um: }\end{array}$} \\
\hline & $>20 \%$ & 1 \\
\hline & $>50 \%$ & 2 \\
\hline & $>100 \%$ & 3 \\
\hline e. & Temperatura retal acima do tisiológico & 1 \\
\hline t. & Salivaçao & 2 \\
\hline \multicolumn{3}{|l|}{$\begin{array}{l}\text { Resposta à } \\
\text { palpaçåăo }\end{array}$} \\
\hline \multirow[t]{3}{*}{ Escola apenas um: } & $\begin{array}{l}\text { Sem azeraçào do comportamento pré- } \\
\text { cinúrgico }\end{array}$ & 0 \\
\hline & Protege/Reage quando tocado & 2 \\
\hline & Protege/Reage antes de ser tocado & 3 \\
\hline \multicolumn{3}{|l|}{ Nivel de atividade } \\
\hline \multirow{5}{*}{$\begin{array}{l}\text { a. Escola apenas } \\
\text { um: }\end{array}$} & Em repouso, dormindo ou semi-consciente & 0 \\
\hline & Em repouso, acordado & 1 \\
\hline & Comendo & 0 \\
\hline & Inquieto (caminhando, levantando e deflando) & 2 \\
\hline & Rolando ou se auto-mutlando & 3 \\
\hline \multicolumn{3}{|l|}{ Postura } \\
\hline & Guardando cu protegendo a area atetada & 2 \\
\hline \multirow{6}{*}{$\begin{array}{l}\text { b. Escolha apenas } \\
\text { um: }\end{array}$} & Deculbito lateral & 0 \\
\hline & Decubito esternal & 1 \\
\hline & Sentado/em estaçao, cabeça para cima & 1 \\
\hline & Em pe, cabeça baixa & 2 \\
\hline & Movimentando & 0 \\
\hline & Postura anormal (posiçăo de reza, arqueado) & 2 \\
\hline \multicolumn{3}{|l|}{ Vocalizaçào } \\
\hline \multirow[t]{4}{*}{ Escola apenas um: } & Năo vocaliza & 0 \\
\hline & Vocaliza quando tocado & 2 \\
\hline & Vocalizaçao intermitente & 2 \\
\hline & Vocalizaçao continua & 3 \\
\hline \multirow{5}{*}{$\begin{array}{l}\text { Estado mental } \\
\text { Escola apenss um: }\end{array}$} & & \\
\hline & Submisso & 0 \\
\hline & Multo amigavel & 1 \\
\hline & Desconfiado & 2 \\
\hline & Agressivo & 3 \\
\hline
\end{tabular}

Escore de zero a 22. Quanto maior a pontuaçăo maior a dor pósoperatória. Adaptado de FIRTH \& HALDANE (1999).

Fonte: Firth \& Haldane (1999). 